\section{Mammakarzinom: EndoPredict erleichtert Therapieentscheidung}

Mit EndoPredict ${ }^{\circledR}$ steht für die Indikation Brustkrebs seit Kurzem ein Multigen-Test der zweiten Generation für Patientinnen mit hormonrezeptorpositivem Tumor zur Verfügung.

Insbesondere bei dieser - mit etwa $65 \%$ größten - Patientinnengruppe reichen die klassischen klinisch-pathologischen Prognosefaktoren meist nicht aus, um eine klare Aussage darüber zu treffen, welche Therapie für eine Patientin optimal ist. Mit EndoPredict werden nun zielgenau die 6 von 10 Fällen identifiziert, die mit einer alleinigen antihormonellen Therapie - und ohne die belastende Chemotherapie - optimal behandelt sind.

Als erster Test seiner Art lässt er sich in den örtlichen Pathologien durchführen und somit über die Kostenträger als ärztliche Leistung abrechnen. Der bei anderen Tests oftmals notwendige Versand an ein Referenzlabor im Ausland entfällt. Nicht zuletzt durch diese logistische Erleichterung kann das Testergebnis binnen 24 Stunden vorliegen. Somit wird das Ergebnis zeitnah von den betreuenden Fachärzten im Kontext aller Untersuchungsergebnisse beurteilt.

Durchgeführt wird diese Diagnostik standardmäßig an fixiertem Gewebe, das durch eine Biopsie oder während einer Operation gewonnen wurde.

Klinisch überprüft wurde der Test im Rahmen zweier breit angelegter Phase-III-Studien der Austrian Breast and Colorectal Cancer Study Group (ABCSG-6 und ABCSG-8). Mit der
Auswertung von insgesamt 12 Genen konnten treffsichere Prognosen über den Verlauf der Erkrankung gegeben werden. Es wurde bestätigt, dass EndoPredict die Patientinnengruppe identifiziert, die unter alleiniger antihormoneller Behandlung eine mehr als 95\%-ige Wahrscheinlichkeit für ein Metastase-freies Überleben von mindestens 10 Jahren erwarten kann [1].

\section{Literatur}

1 Filipits M et al.: Clin Cancer Res 2011;17(18): 6012-6020.

Weitere Informationen bei

Sividon Diagnostics GmbH

Esther Linnenberg

Nattermannallee 1, 50829 Köln

Tel. +49 221 669561-70, Fax -99

linnenberg@sividon.com

\section{Eribulin beim metastasierten Mammakarzinom - auf dem Weg zur Standardtherapie?}

Bislang gibt es beim metastasierten Mammakarzinom keine etablierte Standardtherapie. Die Patientinnen sind in aller Regel bereits mit Anthrazyklin- und Taxanhaltigen Chemotherapieregimen behandelt worden, eine evidenzbasierte Strategie besteht für die metastasierte Situation nach einer solchen Vortherapie aber nicht.

«Liegt die letzte Chemotherapie schon länger zurück, so kann eine Re-Induktion mit den schon früher verabreichten Wirkstoffen versucht werden», erläuterte Professorin Tjoung-Won Park-Simon, Hannover, beim 30. Deutschen Krebskongress in Berlin auf einem Satellitensymposium von Eisai. Möglich ist auch eine Kombinationstherapie, doch sind beide Strategien nach Park-Simon nicht evidenzbasiert.
Als 3. Option nannte die Medizinerin die Behandlung mit neuen Substanzen wie Eribulin $\left(\right.$ Halaven $\left.^{\circledR}\right)$. Der Wirkstoff ist für die Therapie des metastasierten Mammakarzinoms nach Anthrazyklin- und Taxan-Vorbehandlung in den Leitlinien der AGO (Arbeitsgemeinschaft Gynäkologische Onkologie) als ein Mittel der Wahl aufgelistet. «Eribulin ist dabei der einzige Wirkstoff, für den in der Mono-Chemotherapie eine signifikante Verlängerung des Gesamtüberlebens in Studien belegt wurde», berichtete Park-Simon.

Der innovative Hemmer der MikrotubuliDynamik, der zur Gruppe der Halichondrine gehört und sich in seinem Wirkmechanismus grundlegend von den Anthrazyklinen unterscheidet, hat damit aus Sicht der Onkologin das Potenzial, sich als Standard bei der Behandlung des Mammakarzinoms in fortgeschrittenen Therapielinien nach einem Anthrazyklin und einem Taxan zu entwickeln. Diese Einschätzung basiert auf den Ergebnissen der EMBRACE-Studie (EisaiMetastatic Breast Cancer Study Assessing Treatment of Physician's Choice (TPC) Versus Eribulin E738) [1].

\section{Literatur \\ 1 Cortes J et al.: Lancet 2011;377:914-923.}

Weitere Informationen bei

Eisai $\mathrm{GmbH}$

Dr. Cecilia Hanne

Lyoner Straße 36, 60528 Frankfurt

Tel. +4969 66585-74, Fax -25

caecilia_hanne@eisai.net

\title{
PharmaTicker+++ PharmaTicker+++ PharmaTicker+++ PharmaTicker+++
}

DKG. Deutsche Mediziner können sich jetzt für Best of $\mathrm{ASCO}^{\circledR}$ Germany anmelden. Dieser Onkologiekongress findet am 22./23.06.2012 in Frankfurt statt und ist die einzige Post-ASCOVeranstaltung in Deutschland, die die American Society of Clinical Oncology ${ }^{\circledR}$ (ASCO) offiziell lizenziert hat. Das Programm setzt sich aus den besten Vorträgen des Annual Meetings zusammen. Veranstalter ist die Deutsche Krebsgesellschaft e.V. (DKG). Informationen und Registrierung online unter: www.best-of-asco.de.

art tempi communications gmbh

Diana Atanassova, Bianca-Maria Rathay

atanassova@art-tempi.de, rathay@art-tempi.de
DGHO. Die Deutsche Gesellschaft für Hämatologie und Onkologie e.V. (DGHO) bietet Patienten mit Krebs- und Bluterkrankungen unter www.mein-onkopedia.de eine schnelle, transparente und verlässliche Quelle für alle Fragen rund um die Themen Untersuchung, Behandlung, Nebenwirkungen und Nachsorge. Die eingestellten Patientenleitlinien auf diesem Wissensportal sind inhaltlich identisch mit den wissenschaftlich fundierten Leitlinien für Ärzte und werden laufend aktualisiert.

Akzent | Agentur für strategische Kommunikation Kirsten Thellmann, Ulrike Feldhusen k.thellmann@akzent-pr.de, u.feldhusen@akzent-pr.de
Zum 2. Mal seit Einführung des größten europäischen Mammographie-Screening-Programms im Jahr 2005 legt die Kooperationsgemeinschaft Mammographie die Auswertungen der Daten für Deutschland in einem Evaluationsbericht vor. Laut Thorsten Kolterjahn, Vorsitzender des Beirats der Kooperationsgemeinschaft, ist das «Mammographie-Screening-Programm auf einem sehr guten Weg» und demnach «greift die bislang für Früherkennungsmaßnahmen einzigartige Qualitätssicherung».

Pressestelle Kooperationsgemeinschaft Mammographie Corinna Heinrich

cheinrich@koop-mammo.de

\section{KARGER}

(ㅇ) 2012 S. Karger GmbH, Freiburg

Fax +497614520714 Information@Karger.d
www.karger.com 
Mehr als 10 Jahre Zometa ${ }^{\circledR}$ :

\section{Marktführer in der Prävention von skelettalen Ereignissen bei auf den Knochen ausgedehnten Tumorerkrankungen}

Seit der Zulassung von Zometa ${ }^{\circledR}$ (Zoledronat) im Jahr 2002 wurden über 4,6 Millionen Patienten mit Zoledronat behandelt. Damit ist das Medikament weltweit das meistverschriebene Bisphosphonat und in über 100 Ländern zur Prävention von skelettalen Ereignissen bei auf den Knochen ausgedehnten Tumorerkrankungen zugelassen. Über den Knochenschutz hinaus wurden in den vergangenen Jahren zahlreiche klinische Daten erhoben, die Rückschlüsse auf weiterführende Wirkungen durch die monatliche Bisphosphonat-Infusion zeigen.

Bisphosphonate tauchten in der Chemie bereits im 19. Jahrhundert auf, jedoch wurden erst 1968 die ersten biologischen Charakteristika dieser Medikamentengruppe erforscht. Zoledronat wurde schließlich Ende der 80er Jahre von Dr. Knut Jaeggi, einem Chemiker des damaligen Pharmaunternehmens Ciba, synthetisiert. 1993 startete die erste klinische Studie mit dieser Substanz. Zwischenzeitlich profitieren Patienten mit fortgeschrittenen und auf das Skelett ausgedehnten Tumorerkrankungen seit mehr als 10 Jahren von Zoledronat.

Knochenschutz und mehr beim Multiplen Myelom

Eine aktuelle Auswertung der MRC-MyelomaIX-Studie - der mit 1960 Patienten an 121 Zentren bislang größten Myelom-Studie zeigt eine über den Knochenschutz hinaus signifikante Verlängerung des Überlebens um 5,5 Monate im Vergleich zum älteren Standard Clodronat (50,0 vs. 44,5 Monate; $\mathrm{p}=0,04$ ), was einer Verminderung der Mortalität um $16 \%(p=0,0118)$ entspricht [1]. Dabei ist der Vorteil mit Zoledronat vs. Clodronat bereits in den ersten Behandlungsmonaten erkennbar. Zusätzliche Daten einer Post-hoc-Analyse einer doppelblinden Studie zum Vergleich von Zoledronat und Denosumab bei Patienten mit soliden Tumoren zeigen bei Myelom-Patienten einen Vorteil von Zoledronat [2].

Bis heute einziges Amino-Bisphosphonat mit Zulassung beim Prostatakarzinom

Bis heute besitzt Zoledronat zudem als einziges Amino-Bisphosphonat die Zulassung zur Behandlung von Skelettkomplikationen (SREs) bei Patienten mit Prostatakarzinom, die vor allem durch osteoblastische Metastasen verursacht werden. Die Zulassungsstudien zum Prostatakarzinom zeigen zusätzlich zu einem durch Zoledronat um 32\% verringerten SRERisiko (vs. Plazebo; $p=0,025$ ) [3] auch eine gegenüber Plazebo signifikant verringerte Schmerzreduktion [4] sowie eine Normalisierung der Knochenmarker [5]. Auch bei weiteren urologischen Tumoren sowie beim ossär metastasierten Mammakarzinom wird Zoledronat zur Prävention von SREs eingesetzt und zeigt über den Knochenschutz hinaus vielversprechende Ergebnisse [6-8].

Hintergrundinformation: Die Entwicklung von Zometa ${ }^{\circledR}$

Im Jahr 1997 trat Zoledronat in die Phase II/ III zur Behandlung von tumorinduzierter Hy- perkalzämie ein, bereits im darauf folgenden Jahr in die Phase III für die Behandlung von Knochenmetastasen. 2001 erhielt Zometa ${ }^{\circledR}$ die europäische Zulassung zur Behandlung der Hyperkalzämie, seit 2002 ist Zoledronat zur Prävention von tumorinduzierten Skelettkomplikationen Patienten mit fortgeschrittenen, auf das Skelett ausgedehnten, Tumorerkrankungen zugelassen.

\section{Literatur}

1 Morgan GJ et al.: First-line treatment with zoledronic acid as compared with clodronic acid in multiple myeloma (MRC Myeloma IX): a randomised controlled trial. Lancet 2010;376:1989-1999.

2 Henry DG et al.: Randomized, double-blind study of denosumab versus zoledronic acid in the treatment of bone metastases in patients with advanced cancer (excluding breast and prostate cancer) or multiple myeloma. J Clin Oncol 2011;29:1125-1132.

3 Saad F: Clinical benefit of zoledronic acid for the prevention of skeletal complications in advanced prostate cancer. Clin Prostate Cancer 2005;4(1):31-37.

4 Saad F: New research findings on zoledronic acid survival, pain, and anti-tumour effects. Cancer Treat Rev 2008;34(2):183-192.

5 Lipton A et al.: Normalization of bone markers is associated with improved survival in patients with bone metastases from solid tumors and elevated bone resorption receiving zoledronic acid. Cancer 2008; 113(1):193-201.

6 Lipton A et al.: Zoledronic acid delays the onset of skeletal-related events and progression of skeletal disease in patients with advanced renal cell carcinoma. Cancer 2003;98(5):902-909.

7 Zaghloul MS et al.: A prospective, randomized, placebocontrolled trial of zoledronic acid in bony metastatic bladder cancer. Int J Clin Oncol 2010;15(4):382-389.

8 Rosen LS et al.: Zoledronic acid is superior to pamidronate for the treatment of bone metastases in breast carcinoma patients with at least one osteolytic lesion. Cancer 2004;100(1):36-43.

Weitere Informationen bei

Novartis Pharma GmbH

Melitta Zakel

Roonstraße 25, 90429 Nürnberg

melitta.zakel@novartis.com

www.novartisoncology.de

\section{PharmaTicker+++ PharmaTicker+++ PharmaTicker+++ PharmaTicker+++}

ACCURAY. Die effektive Behandlung von Krebserkrankungen erfordert individuelle Therapiemöglichkeiten. Je nach Tumorart, -lage und -größe ist es essentiell, dass die Behandlung auf den jeweiligen Patienten zugeschnitten ist. Hierfür hat ACCURAY strahlentherapeutische Lösungen für die optimale Versorgung von Patienten entwickelt: Sowohl das CyberKnife ${ }^{\circledR}$ als auch das TomoTherapy ${ }^{\circledR}$ System bieten Krebspatienten hochpräzise Behandlungsmethoden für unterschiedliche Tumortypen in allen Körperregionen.

Edelman $\mathrm{GmbH}$

Nadine Gottschling

nadine.gottschling@edelman.com
Teva. AWD.pharma GmbH und CT Arzneimittel $\mathrm{GmbH}$ bilden zusammen innerhalb des Teva-Konzerns in Deutschland die Business Unit Branded. Fundiertes Know-how verbunden mit langjähriger Erfahrung und konsequenter Qualitätsorientierung sind die Basis des Produktbereiches Schmerz mit dem Forceprodukt Katadolon ${ }^{\circledR} \mathrm{S}$ long. Neu aufgenommen in den Schmerzbereich wurde das innovative Produkt Effentora ${ }^{\circledR}$, ein Medikament für die Therapie von Durchbruchschmerzen bei Tumorpatienten, welches das Opioidsortiment sinnvoll ergänzt. BPI Service $\mathrm{GmbH}$ Katharina Piesker piesker@bpi-service.de
Mundipharma. Auf dem Deutschen Schmerzund Palliativtag plädierten Experten im Rahmen eines Symposiums für eine individuelle OpioidAuswahl, wobei Morphin nicht das Mittel der ersten Wahl sei. «Besser geeignet sind z.B. Substanzen wie die Fixkombination aus retardiertem Oxycodon und retardiertem Naloxon $\left(\operatorname{Targin}^{\circledR}\right)$, die sehr gut verträglich ist und die normale Darmfunktion erhält, die durch Opioide sehr häufig eingeschränkt wird», so Prof. Dr. Gerd Mikus, Universitätsklinikum Heidelberg.

Mundipharma $\mathrm{GmbH}$

Felicitas Lanz

felicitas.lanz@mundipharma.de 


\section{nab-Paclitaxel bereichert neue Studienkonzepte beim Mammakarzinom}

Trotz der Einführung zielgerichteter Substanzen bilden wirksame Zytostatika wie die Taxane nach wie vor das Rückgrat der Therapie des Mammakarzinoms. Daher richtet sich die Arzneistoffentwicklung auch auf deren Optimierung, z.B. durch innovative Galenik. Bei nab-Paclitaxel (Abraxane $\left.{ }^{\circledR}\right)$ handelt es sich um die erste tumorgerichtete nab-Chemotherapie. Das an AlbuminNanopartikel gebundene Zytostatikum Paclitaxel macht sich den natürlichen Albumin-Transportweg zunutze und reichert sich selektiv in Tumorzellen an. Hierdurch ergeben sich beim Mammakarzinom eindeutige klinische Vorteile. Diese werden nun in neuen Studienkonzepten wie in der neoadjuvanten GeparSepto-Studie ausgenutzt.

Hohe Effektivität beim metastasierten Mammakarzinom

Die Studie von Gradishar et al. [1] untersuchte randomisiert die Wirksamkeit und Verträglichkeit von nab-Paclitaxel (nab-P) in der Dosierung von $260 \mathrm{mg} / \mathrm{m}^{2}$ alle 3 Wochen verglichen mit konventionellem Paclitaxel in der Standarddosierung von $175 \mathrm{mg} / \mathrm{m}^{2}$ bei 454 Patientinnen mit z.T. vorbehandeltem metastasierten Mammakarzinom (mBC). 60\% der Patientinnen hatten zuvor Anthrazykline erhalten. Das Gesamtansprechen war unter nab-P signifikant besser (33,2 vs. $18,7 \%$, $\mathrm{p}=0,001$ ), wobei sich die signifikante Überlegenheit über alle Subgruppen erstreckte, unabhängig von der Anzahl der Vorbehandlungen und der Metastasenlokalisation, führte Prof. Dr. Michael Untch, Berlin, aus. Sensorische Neuropathien waren unter nab-Paclitaxel häufiger, bildeten sich jedoch schneller zurück als bei konventionellem Paclitaxel. Bei mit nab-P behandelten
Patientinnen trat die Besserung der Neuropathie um mehr als einen Grad auf der CTCAE-Skala median bereits nach 22 Tagen ein, während Patientinnen in der konventionellen Paclitaxel-Gruppe erst nach 79 Tagen eine vergleichbare Besserung der Symptomatik erfuhren. In der Subgruppe der Patientinnen, die nab-P als second- oder furtherline-Therapie erhalten hatten, $(n=267)$ verbesserte nab-P auch das Gesamtïberleben signifikant (56,4 vs. 46,7 Wochen, $\mathrm{p}=0,024$ ).

Die praktische Anwendung der Therapie ist erheblich einfacher, denn im Unterschied zu den konventionellen Taxanen ist nab-P ohne Lösungsvermittler wasserlöslich. Die Infusionszeit ist von 3 Stunden auf 30 Minuten verkürzt, es werden keine speziellen Infusionsbestecke benötigt und die vorher obligate antiallergische Prämedikation entfällt, was laut Untch von großem Vorteil ist.

Wöchentliche Gabe von nab-Paclitaxel noch effektiver

Die randomisierte 4-armige Phase-II-Studie CA024 [2] verglich bei nicht vorbehandelten mBC-Patientinnen wöchentlich appliziertes nab-P in 2 Dosierungen (100 und $150 \mathrm{mg} / \mathrm{m}^{2}$ ) mit der 3-wöchentlichen Gabe von $300 \mathrm{mg} / \mathrm{m}^{2}$ nab-P und mit Docetaxel in der 3-wöchentlichen Standarddosierung von $100 \mathrm{mg} / \mathrm{m}^{2}$. Das beste (74\%) und (median bereits nach 2 Zyklen) rascheste Gesamtansprechen zeigten Patientinnen unter wöchentlich $150 \mathrm{mg} / \mathrm{m}^{2}$ nab-P, erläuterte Prof. Dr. Christian Jackisch, Offenbach. Das Gesamtansprechen war hier signifikant besser als in den anderen 3 Armen, das progressionsfreie Überleben war gegenüber Docetaxel signifikant verlängert (14,6 vs. 7,8 Monate, $\mathrm{p}=0,012)$. Im $150 \mathrm{mg} / \mathrm{m}^{2}$
nab-P-Arm wurde mit 33,8 Monaten das längste mediane Gesamtüberleben (OS) im Vergleich zu den anderen 3 Armen beobachtet [3] (Abb. 1).

Neutropenien vom Grad 4 und Fatigue vom Grad 3 sowie febrile Neutropenien waren unter Docetaxel häufiger, sensorische Neuropathien traten in allen 4 Studiengruppen ähnlich häufig auf. Auch hier bildeten sie sich bei den mit nab-P behandelten Patientinnen rascher zurück als unter Docetaxel.

GeparSepto-Studie untersucht nab-Paclitaxel in der Neoadjuvanz

Bei der neoadjuvanten Therapie des frühen Mammakarzinoms ist eine hohe Effektivität der Therapie gemessen an der pathologischen Komplettremissionsrate (pCR) von entscheidender prognostischer Bedeutung, erläuterte Prof. Jackisch. In die geplante GeparSepto-Studie sollen 1200 Patientinnen mit frühem Mammakarzinom eingeschlossen werden, die eine neoadjuvante Chemotherapie in einer inversen Sequenz erhalten: Im Vergleichsarm bekommen die Patientinnen zuerst konventionelles Paclitaxel gefolgt von 4 Zyklen Epirubicin/Cyclophosphamid (EC) und im experimentellen Arm zuerst nab-P in einer Dosierung von $150 \mathrm{mg} / \mathrm{m}^{2}$ wöchentlich an 12 aufeinander folgenden Wochen (ohne Pause) gefolgt von $4 \times$ EC. Patientinnen mit hormonrezeptorpositiven Tumoren erhalten gleichzeitig zur Chemotherapie eine antihormonelle Therapie und Her2-positive Patientinnen eine Kombination von Trastuzumab und Pertuzumab.

«Diese Studie verbindet verschiedene Therapiestrategien und ist ein Beispiel für den sinnvollen und optimierten Einsatz von Substanzen in der modernen Therapie des Mammakarzinoms», sagte Jackisch.

Dr. Petra Ortner, München

\section{Quelle}

Lunchsymposium «Neue Therapiekonzepte durch die nab-Technologie» am 23.02.2012 im Rahmen des Deutschen Krebskongresses, unterstützt von Celgene GmbH

\section{Referenzen}

1 Gradishar WJ: Expert Opin Pharmacother 2006;7: 1041-1053.

2 Gradishar WJ et al.: JCO 2009;27(22):3611-3619.

3 Gradishar WJ et al.: ASCO Breast Cancer Symposium 2011, San Francisco: poster P5-19-03.

Weitere Informationen bei

Celgene $\mathrm{GmbH}$

Joseph-Wild-Str. 20, 81829 München

Tel. +49 894515190

www.abraxane.de

Abb. 1. Studie nab-Paclitaxel versus Docetaxel: Medianes Gesamtüberleben; modifiziert nach [3] 